\title{
CHEMICAL MODELS OF AGB WINDS
}

\section{CHERCHNEFF}

Physics Department, UMIST

P.O. Box 88

Manchester W601QD, United Kingdom

\begin{abstract}
We present chemical models of the inner circumstellar envelope of a typical carbon-rich AGB star. The effect of pulsation-driven shocks on the gas close to the stellar photosphere is considered. The chemistry of dust condensation nuclei formation is described and applied to the gas layers close to the star. We derive formation yields for polycyclic aromatic hydrocarbon $(\mathrm{PAH})$ species and their dimers and discuss their role in the condensation of dust. Models of the chemistry of silicon- and sulfur-bearing molecules in the inner envelope are also presented.
\end{abstract}

\section{Introduction}

AGB stars are characterized by very extended circumstellar envelopes due to the onset of a strong stellar wind during the thermally-pulsating AGB phase. These envelopes are very rich in molecular species, as evidenced by IR, millimetre, and sub-millimetre observations. They are also fascinating astrophysical environments because the physical parameters of the gas flow change very quickly over the full extent of the wind. Therefore the chemical processes responsible for the formation of molecules are highly dependent on the distance to the star. Close to the photosphere, the gas temperature and density are high and molecular formation is governed by termolecular and bimolecular reactions. These regions are where dust grains condense. Further out in the wind we expect the gas and the solid phases to interact, and molecular formation triggered off by surface chemistry may occur. Also, some molecular species may be depleted from the gas onto dust grains. At large stellar radii (i.e. the outer envelope) the physical conditions are similar to those encountered in molecular clouds, and the resulting chem- 
ical processes are governed mainly by neutral-neutral, ion-molecule, and photodissociation/ionization reactions.

The inner and outer envelopes are closely linked and it is therefore crucial to examine the various processes occuring in the inner region in order to assess the molecular input to the outer envelope and to confront the theoretical molecular abundances to those derived from IR observations. We present models of the carbon chemistry responsible for dust nucleation and models of the silicon and sulfur chemistry in the inner envelope of a typical carbon-rich AGB star.

\section{Description of the inner envelope}

AGB stars are Long-Period Variables with typical pulsation periods of $\sim$ a year. The pulsations result in the formation of shocks just outside the photosphere and these periodic shocks alter dramatically the layers of gas close to the star (Bowen 1988, Cherchneff \& Tielens 1994). Indeed, energy is communicated to the gas by the shock and the gas heats up. The gas then cools via various microscopic processes (molecular dissociation, ionization, recombination, etc...) and via work, i.e., expansion (Willson \& Bowen 1986). As a result, the gas layers close to the photosphere are accelerated upwards, decelerate under the influence of the stellar gravitational field, and eventually fall back towards their initial position. This cycle will repeat itself with the next pulsation and the gas layers will experience oscillatory motions with a period equal to the pulsation period of the star.

In order to investigate the formation of molecules in the inner envelope of a typical C-rich AGB star, we use the standard stellar model of Cherchneff et al. (1993) and study the chemistry occuring in the shocks and in the excursions experienced by the gas. In both cases, the chemistry can be decoupled from the hydrodynamics of the flow because the chemical species considered do not have, as a first approximation, a strong effect on the cooling of the gas and on the dynamics of the wind (Cherchneff et al. 1991, Allain et al. 1996).

\subsection{THE SHOCKS}

The pulsation-driven shocks in AGB stars are low-velocity (typically $20 \mathrm{~km}$ $\mathrm{s}^{-1}$ ), molecular shocks. The cooling in the postshock region is due mainly to the collisional dissociation of molecular hydrogen by atomic hydrogen (Fox $\&$ Wood 1985). Radiative processes are not efficient in cooling the postshock gas because the ionization and excitation in the postshock region are due to collisions with $\mathrm{H}$ atoms and the resultant emission intensities are very low. Therefore we describe the shock profiles assuming the Rankine-Hugoniot jump conditions in the shock front and we consider only $\mathrm{H}_{2}$ dissociation as 
the main cooling process of the postshock gas. We investigate several shock velocities ranging from 20 to $13 \mathrm{~km} \mathrm{~s}^{-1}$. These values correspond to a 20 $\mathrm{km} \mathrm{s}^{-1}$ photospheric shock whose strength is damped as the shock travels through the envelope (Cherchneff \& Tielens 1996).

\subsection{THE EXCURSIONS}

The gas excursions are modelled following a semi-analytical description derived by Bertschinger \& Chevalier (1985). We consider strictly periodic motions, i.e., the parcel of gas accelerated by the shock is moving upwards but fall back to its initial position. Such an assumption is adequate for the regions close to the photosphere where gravity has a strong effect. As the dust formation zone in C-rich AGB stars is believed to correspond to a narrow region at a few stellar radii from the star (Danchi \& Bester 1995), the approximation of periodic motions can apply.

\section{The chemistry of PAHs and PAH dimers}

C-rich AGB stars emit a strong infrared excess due to the presence of dust grains in their extended winds. This excess is better fitted with a mixture of amorphous carbon $(\mathrm{AC})$ and silicon carbide $(\mathrm{SiC})$ particles (Groenewegen 1995) and we investigate the formation processes of $\mathrm{AC}$ grains in the inner, shocked regions of AGB winds. The gas in the inner envelope of C-rich AGB stars is molecular and is rich in $\mathrm{H}_{2}, \mathrm{H}, \mathrm{He}, \mathrm{CO}$, and $\mathrm{C}_{2} \mathrm{H}_{2}$. A large fraction of the available carbon is locked in $\mathrm{CO}$ which survives the hot environment of the inner regions due to its high binding energy. The remaining carbon is in the form of acetylene $\mathrm{C}_{2} \mathrm{H}_{2}$ and this species will react with atomic hydrogen to start an active hydrocarbon chemistry. Such a chemistry is found on Earth in flames rich in acetylene and in many combustion processes involving $\mathrm{C}_{2} \mathrm{H}_{2}$, and we use a chemical scheme based on combustion chemistry to describe the formation of hydrocarbon molecules. As for acetylenic flames, we expect polycyclic aromatic hydrocarbons (PAHs) to form as important intermediates in the dust condensation process (Frenklach \& Feigelson 1989, Cherchneff et al. 1993). Therefore we describe the nucleation of $\mathrm{AC}$ grains by forming large PAHs up to coronene $\left(\mathrm{C}_{24} \mathrm{H}_{12}\right)$ and we study the first step of dust condensation by forming PAH dimers, i.e., non-planar molecules.

The nucleation steps to large PAHs have been described previously (Cherchneff et al. 1993) and consist of various chemical reactions involving hydrocarbons that lead to the closure of the first aromatic ring (i.e., phenyl $\mathrm{C}_{6} \mathrm{H}_{5}$ ). The subsequent growth of aromatic rings to form PAHs can be described by a set of three reactions: addition of $\mathrm{C}_{2} \mathrm{H}_{2}, \mathrm{H}$ abstraction to form a radical, and a second addition of acetylene followed by ring closure. 
These three reactions are important because they determine a gas temperature window of $900-1100 \mathrm{~K}$ in which PAH growth and the formation of large dust precursors is possible (Frenklach \& Wang 1991). Therefore, PAH molecules will be able to form in regions where this temperature window is found. To help meeting this requirement, we apply the inverse greenhouse effect on small PAHs (Cherchneff et al. 1991).

We consider the formation of PAH dimers up to $\mathrm{C}_{24} \mathrm{H}_{11} \mathrm{C}_{24} \mathrm{H}_{11}$. The reaction rates for dimer formation from their $\mathrm{PAH}$ parents are not available and we base our analysis on the reaction of benzene with phenyl

$$
\mathrm{C}_{6} \mathrm{H}_{6}+\mathrm{C}_{6} \mathrm{H}_{5} \rightarrow \mathrm{C}_{6} \mathrm{H}_{5} \mathrm{C}_{6} \mathrm{H}_{5}+\mathrm{H}
$$

for which a rate has been measured by Fahr et al. (1988). We have $k_{1}=$ $5 \times 10^{-13} \exp (-15.7 / \mathrm{RT})\left(\right.$ in $\mathrm{cm}^{3} \mathrm{~mol}^{-1} \mathrm{~s} ; \mathrm{kJ} \mathrm{mol}^{-1}$ where $\mathrm{R}$ is the perfect gas constant and $\mathrm{T}$ the gas temperature). The rates for the formation of PAH dimers larger than biphenyl should be greater than $k_{1}$ because the van der Waals force between molecules increases with their size. This force can then act as a temporary glue which could hold the adduct together while the chemical bond forms. Miller et al. (1984) have estimated the van der Waals forces for several PAHs and they found that the ratio between the force and the number of carbon atoms of the PAH reactants is roughly constant. We use this result to estimate the van der Waals forces for the PAHs involved in this calculation and we scale the reaction rates with respect to the force values.

TABLE 1. Shock Strengths and Preshock Gas Parameters

\begin{tabular}{lllll}
\hline $\begin{array}{l}\text { Radius } \\
\left(\mathrm{R}_{\star}\right)\end{array}$ & $\begin{array}{l}\text { Shock Strength } \\
\left(\mathrm{km} \mathrm{s}^{-1}\right)\end{array}$ & Mach Number & $\begin{array}{l}\text { Gas Temperature } \\
(\mathrm{K})\end{array}$ & $\begin{array}{l}\text { Gas Concentration } \\
\left(\mathrm{cm}^{-3}\right)\end{array}$ \\
\hline 1.3 & 20.0 & 6.2 & 1965 & $1.70 \times 10^{13}$ \\
1.9 & 16.5 & 5.7 & 1565 & $9.84 \times 10^{11}$ \\
2.5 & 14.4 & 5.4 & 1327 & $1.55 \times 10^{11}$ \\
3.1 & 13.0 & 5.2 & 1167 & $4.18 \times 10^{10}$ \\
\hline
\end{tabular}

It is important mentioning that no destruction processes have been considered for dimers in the present calculations and the formation yields presented in Section 4 are then upper limits.

\section{PAH dimer formation yields}

The formation yield of PAHs and PAH dimers is defined as the total number of carbon atoms in PAHs and PAH dimers divided by the total number of carbon atoms initially in the form of hydrocarbons. The yield was calculated for the cycles of shock+excursion described in Section 2. Each shock 
strength corresponds to a certain position in the inner envelope and the correspondance is given in Table 1 as well as relevant preshock gas parameters.

Preliminary results are summarized in Table 2 . In the $20 \mathrm{~km} \mathrm{~s}^{-1}$ shock and in the excursion following, no PAH/PAH dimers can form because the $900-1100 \mathrm{~K}$ temperature window necessary for the growth of PAHs cannot be reached in the flow. PAH formation starts further out at radii of $\sim 2 R_{\star}$, and the yields range between $10^{-5}-10^{-4}$ at $\mathrm{r} \geq 2 R_{\star}$. The yields for the dimers follow the same trend as for PAHs but are three orders of magnitude smaller.

TABLE 2. PAH and PAH Dimer Formation Yields

\begin{tabular}{llll}
\hline $\begin{array}{l}\text { Radius } \\
\left(\mathrm{R}_{\star}\right)\end{array}$ & $\begin{array}{l}\text { Shock Strength } \\
\left(\mathrm{km} \mathrm{s}^{1}\right)\end{array}$ & PAH Yield & PAH Dimer Yield \\
\hline 1.3 & 20.0 & 0 & 0 \\
1.9 & 16.5 & $3 \times 10^{-5}$ & $10^{-8}$ \\
2.5 & 14.4 & $7 \times 10^{-5}$ & $2 \times 10^{-8}$ \\
3.1 & 13.0 & $7 \times 10^{-5}$ & $2 \times 10^{-8}$ \\
\hline
\end{tabular}

We have not considered the destruction of PAH dimers, although these compounds will be undoubtedly destroyed in the shocks. However, the results show that what happens in the shocks does not have an impact on what happens in the excursions following. The gas has no time to reach equilibrium in the postshock region but does so at the beginning of each excursion because the time scales are much longer. Therefore, we expect the dimers to be destroyed in the shocks but to reform in the excursions and to be gradually expelled in the flow.

Dust contents of C-rich AGB envelopes have been derived by Knapp (1985) using long-wavelength infrared excesses and assumed models for the dust. Knapp obtained an average dust-to-gas ratio of $5.2 \times 10^{-13}$. Then assuming a population of dust grains with mean radius of $a=500 \AA$, the ratio of the number of $\mathrm{C}$ atoms in dust grains to the number of $\mathrm{C}$ atoms in acetylene is $\sim 5 \times 10^{-9}$. This value is in very good agreement with the PAH dimer formation yields found in our calculations and support the fact that PAH dimers can be considered as condensation nuclei in the dust condensation process.

\section{Silicon and sulfur chemistry}

Infrared observations of the inner envelope of IRC +10216 have shown that many molecules are present in the inner region (Keady \& Ridgway 1993). 
These species are formed under thermal equilibrium in the stellar photosphere and are then expelled with the flow in the inner shocked regions. Some of these molecules travel through the envelope and get photodissociated by the interstellar UV radiation field penetrating the outer parts of the wind. These species are often referred as "parent molecules" as they are coming from the deep layers of the wind. Among these chemical species are silicon sulfide $\mathrm{SiS}$ and silicon oxyde SiO. Their abundances in the inner envelope derived from IR measurements (Keady \& Ridgway 1993) are very different from the TE abundances they have in the stellar photosphere. Another molecule of interest is cyclic $\mathrm{SiC}_{2}$ which has been observed at millimetre wavelengths in the outer envelope (Lucas 1995) and which shows to be present in the inner envelope as well. Using the model for the inner regions of a typical C-rich star described in Section 2, we have studied the chemistry of silicon and sulfur to try and reproduce observations. Preliminary results are discussed here and the full calculations will be presented elsewhere (Willacy \& Cherchneff 1996).

A first result shows that $\mathrm{SiS}$ and $\mathrm{SiO}$ are destroyed in the shock / excursion cycles close to the photosphere but that they gradually reform as the shocks move to larger radii. We expect their abundances to reach the observed values at radii $\geq 3 R_{\star}$. Methane $\mathrm{CH}_{4}$ is present in the photosphere but Keady \& Ridgway (1993) have shown that they could not reproduce the methane IR lines assuming a methane distribution close to the star. Our results show low abundances of $\mathrm{CH}_{4}$ close to the photosphere and destruction of the molecule in the shock/excursion cycles at $2.5 R_{\star}$. This supports the idea that the formation of methane takes place at larger radii and may be triggered off by surface reactions on dust grains. A similar conclusion is reached for silane $\mathrm{SiH}_{4}$ which is however not initially present in the photosphere and does not appear to form in the inner gas. Cyclic $\mathrm{SiC}_{2}$ is found overabundant in our calculations with a value 3 orders of magnitude larger than that derived from observations. It is possible though that cyclic $\mathrm{SiC}_{2}$ is involved in the condensation of solid $\mathrm{SiC}$ via processes similar to those observed in the laser-induced pyrolysis of silane and acetylene (Lihrmann $\&$ Cauchetier 1994). Finally, several oxygen-bearing species $(\mathrm{O}, \mathrm{OH})$ are formed in the shocks between 1.3 and $3.1 R_{\star}$ and should be included in future works in the chemical scheme describing dust condensation. Indeed, $\mathrm{O}$ and $\mathrm{OH}$ can stop aromatic growth via oxidation or create new radical sites on PAHs and then favour the formation of larger aromatic species.

\section{References}

Allain, T., Sedlmayr, E., Leach, S. 1996, A\&A 305, 616

Bertschinger, E., Chevalier, R.A. 1985, ApJ 299, 167

Bowen, G.H. 1988, ApJ 329, 299 
Cherchneff, I., Barker, J.R., Tielens, A.G.G.M. 1991, ApJ 377, 541

Cherchneff, I., Barker, J.R., Tielens, A.G.G.M. 1993, ApJ 413, 445

Cherchneff, I., Tielens, A.G.G.M. 1994, in Circumstellar Media in Late Stages of Stellar Evolution, eds. R. Clegg, I. Stevens, W.P.S. Meikle, (Cambridge University Press), p. 232

Cherchneff, I., Tielens, A.G.G.M. 1996, in preparation

Danchi, W.C., Bester, M. 1995, Astrophys. Space Sci. 224, 339

Fahr, A., Mallard, W.G., Stein, S.E. 1988, in Symposium (Int.) on Combustion, 21, The Combustion Institute, Pittsburgh, p. 825

Fox, M.W., Wood, P.R. 1985, ApJ 297, 455

Frenklach, M., Feigelson, E.D. 1989, ApJ 341, 372

Frenklach, M., Wang, H. 1991, in Symposium (Int.) on Combustion, 23, The Combustion Institute, Pittsburgh, p. 1559

Groenewegen, M.A.T. 1995, A\&A 293, 463

Keady, J.J., Ridgway, S.T. 1993, ApJ 406, 199

Knapp, G.R. 1985, ApJ 293, 273

Lihrmann, J.M., Cauchetier, M. 1994, J. of European Ceramic Soc. 13, 41

Lucas, R. 1995, Astrophys. Space Sci. 224, 380

Miller, J.H., Mallard, W.G., Smyth, K.C. 1984, J. Phys. Chem. 88, 4963

Willson, L.A., Bowen, G.H. 1986, in Cool Stars, Stellar Systems, and the Sun, Vol. 254, (Berlin: Springer), p. 385

Willacy, K., Cherchneff, I. 1996, in preparation

\section{Discussion}

Langhoff: A key step in the combustion process is the abstraction of hydrogen. In normal combustion processes in the presence of air, oxygen species are primarily responsible for abstraction. In your model, which does not presently include $\mathrm{O}$ or $\mathrm{OH}$, what reactions provide for hydrogen abstraction?

Cherchneff: The reaction responsible for $\mathrm{H}$ abstraction in our model involves one $\mathrm{PAH}$ with an $\mathrm{H}$ atom to give a $\mathrm{PAH}$ radical and $\mathrm{H}_{2}$.

Rawlings: As we know, carbon dust is seen to form in astrophysical regions where $\mathrm{C}_{2} \mathrm{H}_{2}$ cannot be present, either as a result of photodissociation or else because hydrogen is very deficient. Have you considered chemical pathways to $\mathrm{PAH}$ formation which do not involve the $\mathrm{C}_{2} \mathrm{H}_{2}$ monomer?

Cherchneff: In hydrogen deficient environments where $\mathrm{C}_{2} \mathrm{H}_{2}$ is not present, the intermediates involved in the aromatic nucleation are expected to be pure carbon chains such as $\mathrm{C}_{2}, \mathrm{C}_{3}, \mathrm{C}_{10}$ etc. Indeed, you can form these long chains around Wolf-Rayet or R CrB stars.

Henning: TiC may be the precursor of the solid carbon formation because such particles are found embedded in graphite found in interplanetary dust particles. That is not the case for SiC.

Cherchneff: Definitely, you may have candidates for condensation nuclei other than $\mathrm{SiC}$ if you consider aromatic growth over the surface of a particulate. We have not yet included TiC formation in our model.

Zare: Might particulates play a catalytic role in the formation of PAHs?

Cherchneff: It may be possible but such a process has not been identified yet in flames. 


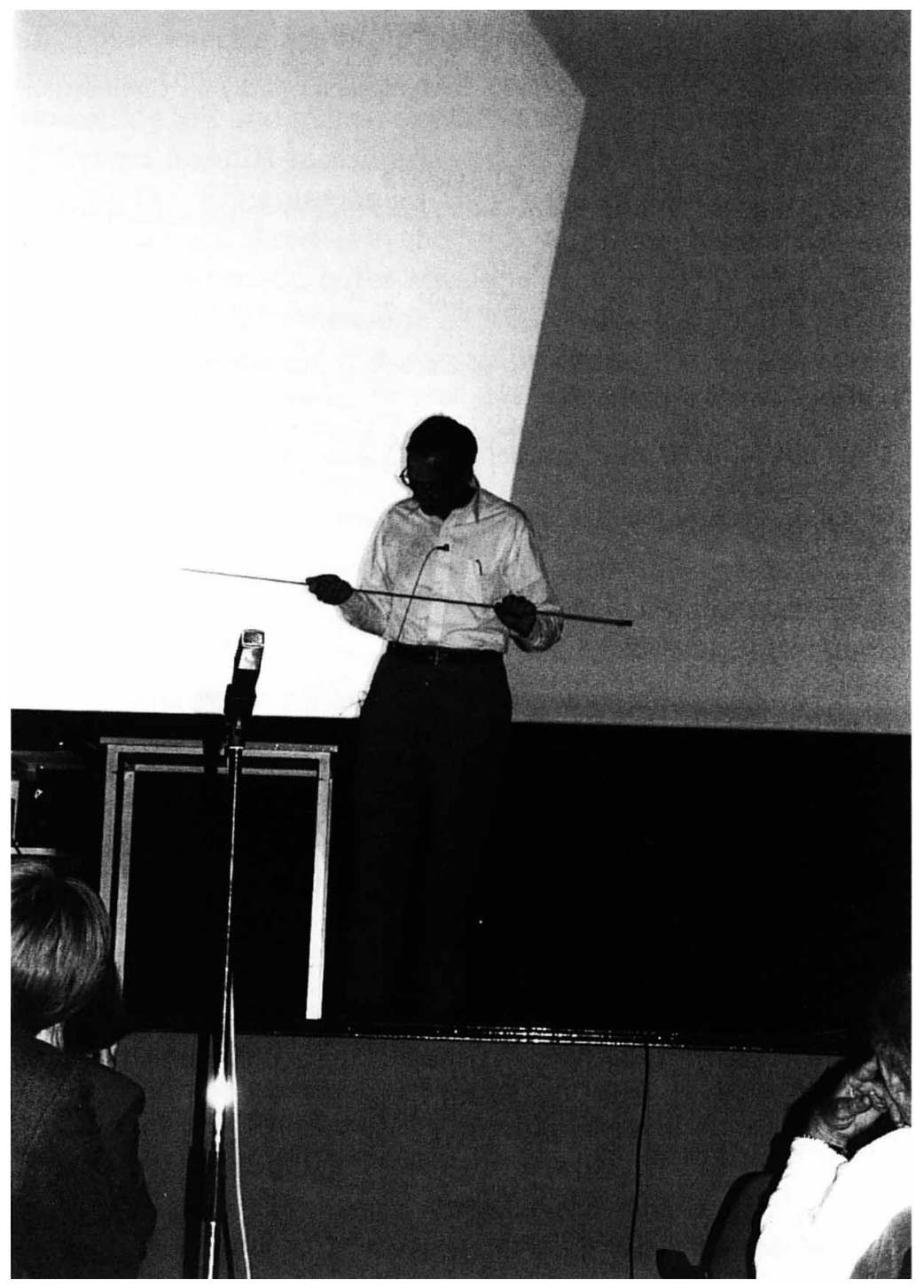

